\title{
LE CANNIBALISME CHEZ LES TRUITES : RÉALITÉ OU LÉGENDE ?
}

\author{
Par J.-A. LESTAGE \\ Vice-Président des Naturalistes Belges, \\ Directeur du Laboratoire de Recherches hydrobiologiques.
}

Commençons par bien poser le problème, car il est d'importance. La mise en valeur d'un biotope salmonidien est sous la dépendance de deux séries de facteurs : il y a les facteurs positifs, dits "facteurs de valorisation ", et les facteurs négatifs, dits "facteurs de dévalorisation ". Rien de compliqué à ceci.

Parmi les seconds facteurs, que nos lecteurs connaissent évidemment, il y avait celui-ci : "Les Truites se mangent entre elles ". Ce facteur n'était nié par personne. LEGER a écrit à ce sujet : " la destruction autophagique des alevins... est d'autant plus grande que les sujets préexistants sont plus nombreux " (I). Un élève du savant professeur cité ci-dessus, .STankovitch, dit que " la Truite paraît manger peu de Poissons dans les cours d'eau alpins " (en note : " sans doute pour la bonne raison qu'elle n'en trouve point ou très peu "). Cependant, l'auteur se range à l'opinion de ceux " qui prétendent que la Truite de grosse taille fait une chasse impitoyable aux petits Poissons, même à ceux de sa propre espèce " (2).

Je pourrais trouver d'autres citations, mais elles concordent, sans aucun doute, avec les précédentes. Il est donc indéniable que le cannibalisme truttien existe, et qu'il constiţue un facteur dévalorisant dont il faut tenir compte pour établir, aussi exactement que possible, le rendement d'un cours d'eau sur la base des déversements effectués et du déchet provoqué par le dit facteur.

Pendant plusieurs années, j'acceptai cette " loi ". Pourtant, au fur et a mesure de mes explorations pour l'étude de la capacité ichtyogénique de centaines de ruisseaux et rivières, au cours desquelles je multipliai les autopsies de Truites fario, en vue de montrer aux agents forestiers quels sont les divers organismes constituant la nourriture de ces Poissons pendant

(1) LfGgr (L.). - Annales de l'Université de Grenoble, XXII, 3, rg1o, p. 31 .

(2) Stangovitch. - Travaux du Laboratoire de l'Institut de Pisciculture de l'Univer. sité de Grenoble, rg20. 
toute l'annẹe, je constatais, non sans curiosité, que je trouvais dans les Truifes des Vairons, des Loches, des Chabots, même des Ecrevisses, mais " jamais rien qui fat d'origine salmonidienne ! ! " Les fario belges feraientelles exception ? Elles sont nettement ichtyophages, sans être même " grosses ", mais pas du tout cannibales !

Cette constatation m'incita à ouvrir une enquête internationale dans la revue Pêche et Pisciculture qui a des lecteurs un peu partout.

En Septembre 1933 , je posai ouvertement la question : - “ En eau libre, les Truites se mangent-elles entre elles ? (I). Il semble que non, si l'on se base sur ce qu'en dit Vougn (2), en Suisse, et divers autres auteurs américains et canadiens dont on verra les opinions dans mon article cité plus haut.

Les premières réponses me vinrent évidemment de Belgique. Celle de M. Mrtzdorf, membre du Comité directeur de la Société Centrale pour la Protection de la Pêche Fluviale, un merveilleux pêcheur de Truites et un amateur des questions hydrobiologiques, fut négative, de même que d'autres émanant de pêcheurs et d'inspecteurs forestiers.

M. M. dE Sérys;-Longhamps, Secrétaire perpétuel de l'Académie, Directeur de l'Institut zoologique Torley-Rousseau, Professeur à l'Université de Bruxelles, pecheur de Truites, me fit savoir qu'il " avait contrôlé plus de 50.000 Truites en près de 50 ans, capturées par lui dans une seule rivière, le Bocq. Il a trouvé des Chabots, petits Brochets, Ammociètes, " mais jamais rien qui fut même suspect de représenter une Truitelle... " (3).

Passons aux investigations faites à l'étranger. En Bohême, Vaclav Dran (4) étudia les contenus stomacaux de 300 fario capturées dans divers ruisseaux de la Moldavie, nota exactement ce qu'il y découvrit comme organismes de tous ordres, notamment $\times 7$. fois sur roo des Poissons (Chabots et Vairons), mais ne trouva rien d'origine salmonidienne, ce qui fit dire au $D^{r}$ ANDrk : " c'est là un fait qui vient à l'appui de la thèse du cannibalisme seulement occasionnel de la Truite " (5).

En Suisse, le $D^{x}$ Emile ANDnḱ, un nom que nos lecteurs ont en haute estime, reconnut a son tour que "cette question a une grande portée "; qu'il faut, pour la résoudre, " des enquêtes consciencieuses et méthodiques... ". Los démonstrations citées dans Pêche et Pisciculture et celle de Vaclav Drcr ne le contentaient pas. "Nous ne pouvons pas, néanmoins, nous empécher de croire que le cannibalisme entre en ligne de compte pour expliquer le déchet que l'on constate toujours dans les ruisseaux d'élevage

(I) Lesstage (J.-A.). - L'adelphophagie chez les Truites. - Peche et Pisciculture, Bruxe:les, $n^{\circ}$ 9, 1933, p. 231 .

(2) Vouga. - Bulletin Suisse de Peche et Pisciculture, Neuchatel, n॰ 8, 1933, p. 126.

(3) De SkLYs-Lonchamps (M.). - Bulletin de l'Institut Zoologique Torley-Rousseau, I, I, Ig27, et Peche et Pisciculture, 1933, n* 10.

(4) Drax. - Archiv für Hydrobiologie, Bd. XXVII, rg34, p. 63̇.

(5) ANDnE (Dr E.). - Peche et Pisciculture, 1935, no 3, p. 54. 
(de 30 à $90 \%$ dans la région que nous connaissons), d'autant plus que, dans ces ruisseaux, il arrive souvent de trouver entre les truitelles des différences de taille allant du simple au triple... ". Le savant professeur de Genève conservait donc un certain scepticisme, et il annonçait que, de son côté, il allait faire des recherches personnelles ( 1 ).

En 1935, il nous donna une étude basée sur l'examen de 113 fario de 80 à 45o gr. provenant du Lyssbach. Les contenus stomacaux contiennent de tout, sauf des Truitelles et même des ceufs. Lisons avec soin la conclusion de cette étude : " C'est souvent au cannibalisme, peut-être exclusivement au cannibalisme, que lẹ pisciculteurs attribuent le déchet, parfois énormes, que l'on déplore dans les bassins, canaux, ruisseaux où l'on élève des alevins de Truite jusqu'aux stades de sömmerling ou de jahrling. Le fait qu'il arrive fréquemment de trouver dans ces élevages des Truitelles dont les dimensions vont du simple au triple semble justifier cette manière de voir. Cependant, il est vraisemblable, même peut-être hors de doute, qu'il y a d'autres causes à ce déchet..." (2).

En $19^{36}$, le même auteur publia une nouvelle étude basée sur l'examen de 225 fario. Il trouva des Loches ( 3 fois), des Chabots (2 fois), un Vairon (I fois), des restes indéterminables ( 3 fois), n'appartenant cependant pas à des Truites ; mais rien qui fût d'origine salmonidienne, bien que le ruisseau eût reçu un déversement de 10.000 alevins. Lisons encore la conclusion finale : " pour trancher cette question..., il faut, non pas des anecdotes, mais des chiffres et encore des chiffres $"(3)$.

En $19^{3} 7$, le $D^{r}$ André, jugeant que la question du cannibalisme truttien méritait d'être signalée au plus grand nombre de lecteurs et de chercheurs possible, publia le même article dans le premier numéro d'une nouvelle revue suisse (4).

Entre temps, j'ai reçu de divers lecteurs de Pêche et Pisciculture une abondante documentation concluant au rejet de la théorie du cannibalisme généralisé des Truites en eau libre (5).

Voilà le côté positif de la question, car il y a aussi un côté négatif, celui des procannibalistes.

L'argumentation ne repose pas sur des bases identiques comme on va le voir, bien que je ne puisse citer en entier, ici, les textes qui m'ont été envoyés.

Différencions tout d'abord ceux qui semblent croire au cannibalisme, mais qui l'excluent pourtant pour la raison qu' "il est difficile, dans la nature, parmi les Truites libres, que les plus grosses de ces dernières trou-

(1) ANDre (Dr E.). - Peche et Pisciculture, $1935, n^{\circ} 3$.

(2) André ( $\mathrm{D}^{\mathrm{r}}$ E.). - Bulletin de la Societé Centrale d'Aquiculture et de Peche, Paris, 1935.

(3) ANDRE (Dr E.), - Ibid., rg36.

(4) ANdre ( $\mathrm{D}^{\mathrm{r}} \mathrm{E}$.). - Le Pecheur Suisse, Lausanne, $1937, \mathrm{n}^{\circ} \mathrm{x}$.

(5) On la trouvera dans Peche et Pisciculture, rg36-1937. 
vent dans leur entourage des truitelles dont elles pourraient faire leur proie. D'ordinaire, les unes et les autres ont des habitats différents... Les plus groseses ropoussent ainsi les plus petites, qui sont obligées d'aller se giter en des lieux, ruisselets et zones d'amont, où les autres ne vont pas. Aussi ces dernières, dans les eaux libres, ont-elles rarement l'occasion d'a voir des truitelles à consommer. Il y a évitement mutuel... ".

On constatera que cette cénobiose de Truites de tailles différentes n'existerait pas selon le $D^{r}$ Rouie, qui voulut bien m'écrire ces lignes, alors qu'elle existe suivant le $D^{r} E$. Andre, et c'est aussi l'opinion de nombre de. nos pêcheurs spécialistes. L'un d'eux, président d'une puissante Société de pêcheurs liégeois, écrivait même récemment que, si l'on trouve surtout des Chabots, Vairons, etc., dans l'estomac de Truites, et peu de truitelles, " c'est, à mon avis, parce que la petite Truite est plus agile, plus rapide, plus méfiante que les autres poissons cités plus haut " (I). Je crois que cette lourdeur des Truites, opposée à la rapidité des truitelles, est à prouver. Cependant, le dit auteur affirme que "la. Truite est un cannibale parfait " !! Des preuves ? Elles ont la même valeur que celles qui me furent données par nombre de pêcheurs de tous pays qui sont aussi affirmatifs, parce que, au moment où ils retiraient de l'eau une Truite prise à l'hameçon, une autre se précipitait sur la captive, d'où " doublé de Truite " pour l'heureux pêcheur. Evidemment, il n'est pas question, ici, de cannibalisme généralisé, facteur de dévalorisation dans les milieux salmonicoles libres, le seul qui nous intíresse.

J'ai, à plusieurs reprises, connu des cas où l'autopsie révéla la présence de truitelles dans l'èstomac d'autres Truites. Je citerai seulement une référence due à $M$. Vaucherot, vice-président de l'Union départementale des Sociétés de pêche du Doubs. Il fit examiner pendant 4 ans envinon 6.00o Truites de 250 à 1.500 grammes. "Jamais un alevin ou une truitelle n'a été trouvé... Ce n'est que tout à fait exceptionnellement que l'on a rencontré quelques alevins ou truitelles qui étaient physiquement déficients (alevins à grosse tête ou déformés congénitaux) ". Et voici ma conclusion : " · la Truite montre par là qu'elle est carnassière, ce que pereonne ne conteste, mais non cannibale... La peur du cannibalisme ne doit donc pas retenir l'alevineur " (2). Ce qu'il convient aussi de retenir de ceci, c'est qu'il y a, dans un mêrne biotope, des alevins, des truitelles et des Truites allant de la demi-livre: au kilo et demi, donc une cénobiose réelle entre Truites de toutes tailles. Est-ce la localisation différente des petites et des grosses qui fait obstacle au cannibalisme?

Est-il impossible que, au cours de leurs chasses, souvent même lointaines, où les Truites rencontrent Vairons, Chabots, Brochetons, Ammocètes, Grenouilles, etc... elles ne soient jamais en contact avec des alevins et des

(1) Cfr. - Ourthe et Amblève, 1936, Juillet, no 7, p. 2, $\infty$, I.

(a) Cfr. - Peche et Pisciculture, Ig35, n॰ 8, p. 180 . 
Truitelles ? C'est possible, mais c'est difficile à croire, d'autant plus qu'il m'est arrivé maintes fois, au cours de mes recherches sitométriques dar des ruisseaux et ruisselets de voir fuir devant mon filet des Truites de tailles bien différentes.

Je citerai enfin une opinion qui affirme que, même si des autopsies, si nombreuses soient-elles, ne font pas découvrir des cas de cannibalisme, celui-ci n'en existe pas moins. Libre à qui voudra de se baser sur une simple affirmation, et non sur les résultats d'autopsies très nombreuses (environ roo.00o, en totalisant celles que je connais de Belgique, de France, de Suisse, de Bohême, d'Allemagne, d'Amérique), pour reconnaître que, si l'on peut parfaitement admettre un cannibalisme occasionnel, il semble bien que la thèse d'un cannibalisme généralisé normal soit, au moins, très sujette à caution. On en arrive, par des recherches personnelles, à des conversions aussi heureuses que celles de l'éminent zoologiste suisse, le $D^{r}$ ANDRé, et à des conclusions dont l'énoncé est absolument antithétique à la croyance ancienne, à savoir : le cannibalisme truttieu ne serait plus un facteur intervenant dans le potentiel ichtyogénique des eaux salmonicoles libres, et il faut chercher autre part la cause du déchet $x$ qui survient.

Ces recherches, dont nul ne peut méconnaître l'importance économique, ii importe de les continuer, même officiellement. Notre Gouvernement s'y intéresse de façon particulière. Sur l'initiative précieuse de M. l'Inspecteur Générall Pérau, l'Administration des Eaux et Forêts a demandé à ses agents de faire capturer sur les frayères un certain nombre de fario, pour que l'étude des contenus stomacaux et intestinaux puisse démontrer s'il y a cannibalisme ou non, si des oufs de Truites sont détruits par les géniteurs montants ou descendants, s'il y a anorexie en période de fraye, anorexie qui serait suivie d'une boulimie nécessitant une alimentation copieuse au dépens de tout ce qui peut être saisi par les affamées. Les résultats des premières recherches que j'ai effectuées par ordre du Gouvernement, en Mars 1936 (r), sont entièrement négatifs.

Un correspondant de la Pêche Illustrée (Octobre 1936) demandait que " des investigations soient faites dans d'autres ruisseaux que ceux où le $D^{r}$ ANDRÉ avait fait les siennes ". Compte-t-il pour rien les recherches de Vaclar et celles que j'ái effectuées dans des centaines de ruisseaux belges, et celles de tous ceux qui, en France et en Belgique, ont bien voulu me prêter leur précieux concours? Il ne tient qu'à lui et à tous ceux que la question intéresse de faire les mêmes expériences autour d'eux.

(1) Ce travail est en cours d'impression dans les Annales de la Société royale zoologique. 